\title{
Transaction
}

\section{Super High Water-Repellent Treatments of Various Kinds of Cloths Using Sol/Gel Method}

\author{
Mitsuru Mizuno $^{* 1}$, Yuri Chaya ${ }^{* 2}$, Tsutomu Takagi*1 ${ }^{* 1}$ Kazumasa Hirogaki ${ }^{* 1}$, and Teruo Hori*1 \\ ${ }^{* 1}$ Fiber Amenity Engineering Course, Graduate School of Engineering, University of Fukui, \\ 3-9-1 Bunkyo, Fukui 910-8057, Japan \\ ${ }^{* 2}$ Hokuriku Environment Service Co., Ltd., 1-207, Saburomaru, Fukui 910-0036, Japan
}

\begin{abstract}
Using mixtures of two kinds of silane compounds, tetraethoxysilane and triethoxy-1H,1H,2H,2Htridecafluoro-n-octylsilane (commercial name: Fluowet FS600, Clariant Co., Ltd. ), super high water-repellent treatments were investigated on several kinds of fabrics. First, the mixtures with different mixing ratio were adjusted to pH 4.0 by adding acetic acid and they were aged for several hours under agitation to be proceeded condensation reaction of silane compounds. Then, the fabrics were immersed in such prepared silane mixtures. The excess of the mixtures on the fabrics were removed and then the fabrics were heated to $150^{\circ} \mathrm{C}$ to fix the silane compounds on the fabrics surface. The reaction of silane compounds were confirmed using FT-IR and EDX analysis. Very high contact angle of water droplet was observed even after enough washing.
\end{abstract}

(Received 24 November, 2011; Accepted 27 December, 2011)

\section{Introduction}

Water-repellent treatment is one of the most important technologies in textile industries. Several kinds of methods have been applied for this purpose [1]. The most common method is coating or laminating of polymer resins containing water-repellent agents such as silicon or fluorine compounds on the clothes [2-4]. In recent years, in water-repellent evaluation, it appears the thing over $150^{\circ}$ of contact angle of water, we came to call these "super high water repellent" $[5,6]$.

On the other hand, researches using a reactive water repellent agent have been reported in recent years. Some of them are use reactive silane coupling agent. The mixture of perfluorooctylsilane and PDMSU was applied onto the cotton cloth [7] and cotton and PET were processed with oligomeric fluoroalkylating agents having blocked isocyanate group [8]. Moreover, there are researches fixing a fluorinated vinyl monomer by an electron beam grafting process [9] or vapor-depositing water-repellent silane monomer on to plasma treated PET [10]. Furthermore, using particulates were studied. Condensated particulates of hydrolyzed tetraethoxysilane and fluoroalkylsilane in ammoniacal alkaline were coated onto several fabrics [11], the silica nanoparticle of superhydrophobic surface was laminated using the layer-bylayer method [12] and layer which gave the self-assembly function was coated with polystyrene beads and silica nanoparticle [13].
Although it inquires by various methods, the perfluoro type of polymers is most used in spite of being inferior compared with the former in hydrofuge and durability performance by the PFOA, just because what still exceeds this practically does not exist.

As a new technique of functionalisation of textiles great attention is focused on so-called sol/gel methods [14]. In this paper a novel super water-repellent treatment method was investigated by means of sol/gel method using a mixture of tetraethoxysilane and triethoxy- $1 \mathrm{H}, 1 \mathrm{H}$, 2H,2H-tridecafluoro-n-octylsilane(Fluowet ${ }^{\circledR}$ FS600).

\section{Experimental}

\subsection{Chemicals and Textile Materials}

Tetraethoxysilane (TEOS) and triethoxy-1H,1H,2H, 2H-tridecafluoro-n-octylsilane (Fluowet ${ }^{\circledR} \mathrm{FS} 600$ ) were purchased from Shinetsu Chemicals Co., Ltd. and Clariant Co., Ltd., respectively. The chemical structures of these compounds are shown in Fig. 1. The other chemicals acetone, ethanol and acetic acid were purchased from Wako Chemicals Industry Co., Ltd. and used without further purification.

Cotton, polyester (PET) and PET/Cotton (65/35) fabrics were purchased from Shikisen-sha Co., Ltd. and Vinylon was kindly supplied from Kuraray Co., Ltd.. These clothes were rinsed by usual methods before use.

\subsection{Preparation of sol-gel solution}

Six kinds of TEOS and Fluowet FS600 mixtures 


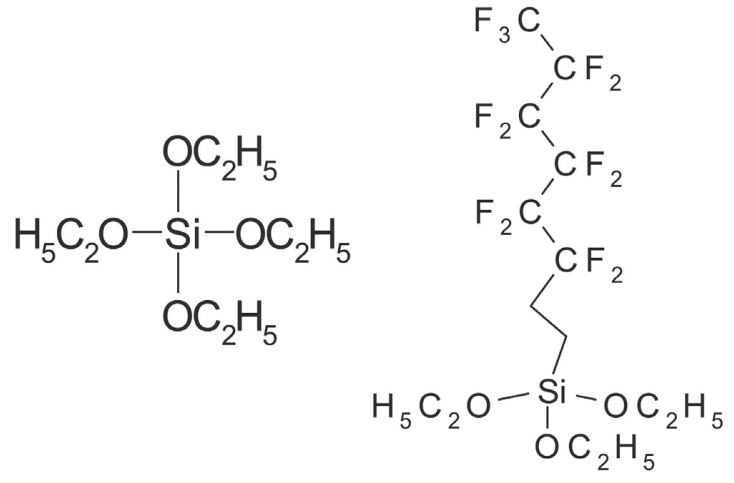

Fig. 1 Structure of silane compounds used for waterrepellent treatment. The left compound is tetraethoxysilane and the right one is triethoxy$1 \mathrm{H}, 1 \mathrm{H}, 2 \mathrm{H}, 2 \mathrm{H}$-tridecafluoro-n-octylsilane.

were prepared. Molar ratio of TEOS and Fluowet FS600 in the mixture was $10: 1,5: 1,1: 1,1: 5,1: 10$ and 1 : 100. Each mixture was diluted with 57 times mol of water and with same mol of ethanol. The atomic content of $\mathrm{Si}$ in every solution was $0.54 \%$.

\subsection{Sol-gel treatments}

$\mathrm{pH}$ of the each mixed solution of Fluowet and TEOS containing a certain amount of water and ethanol was controlled at 4.0 by adding acetic acid. After then the solution was agitated with magnetic stirrer at $400 \mathrm{rpm}$ for 2 days to get the condensate (sol) of TEOS and Fluowet FS 600 . The viscosity of the aged solution was a little increased. The FT-IR of the solution before and after agitation was measured to check the condensation reaction between the Fluowet and TEOS molecules.

The fabric samples cut in the size of $20 \times 60 \mathrm{~mm}$. The PET, cotton and PET/cotton fabrics were washed with acetone to remove the impurities and rinsed with water. Vinylon fabric was washed using an aqueous solution of surfactant Demol N (Kao Co., Ltd.) and rinsed with water. They were then dried with an oven. The rinsed fabrics were immersed in the aged TEOS/Fluowet solution for a few minutes and picked up to dry in atmospheric condition. These fabrics were then treated at $150^{\circ} \mathrm{C}$ in an oven for 30 minutes. The weight change of the fabrics before and after sol/gel treatment measured.

The treated fabrics were then rinsed with methanol under ultrasonic wave irradiation to remove unreacted silane compounds. The fabrics were rinsed well with water and dried at $150^{\circ} \mathrm{C}$ for 15 minutes. The dried fabrics were again weighted to evaluate the amounts of unreacted silane compound.

\subsection{Analysis}

Water-repellent properties were estimated before and after washing using contact angle measurement apparatus
Easy Drop (KRÜSS GmbH). The analysis of the surface of treated fabrics was done by means of FTIR-ATR method (FT-IR 4100 type A, ATR-500/M, Joel Co., Ltd.) and Energy-Disperse X-ray Analyser (EDX, JSM-63901, Joel Co., Ltd.).

\subsection{Evaluation of sol/gel treatment}

Contact angle of water droplet on the fabrics was measured before and after sol/gel treatment. $10 \mu \mathrm{l}$ pure water was dropped on the fabric and the changing of the contact angle of the droplet was observed for 60 minutes. The five measurements after 60 minutes were done to estimate the average values of contact angles.

The contact angle measurements were done also after keeping in atmosphere for 1 week. Before measurements the fabrics were washed again and dried at $150^{\circ} \mathrm{C}$

\section{Results and discussion}

\subsection{Water repellent property of sol/gel treated fabrics}

Fig. 2(a), (b), (c) and (d) show the contact angle of the blank and treated PET, cotton, PET/cotton and vinylon fabrics before and after sol/gel silane treatments by changing Fluowet content, respectively. Estimation was carried out after enough washing. The shapes of the water droplet are also observed and shown in the right side of each figure.

In the cases of cotton, PET/cotton and vinylon the
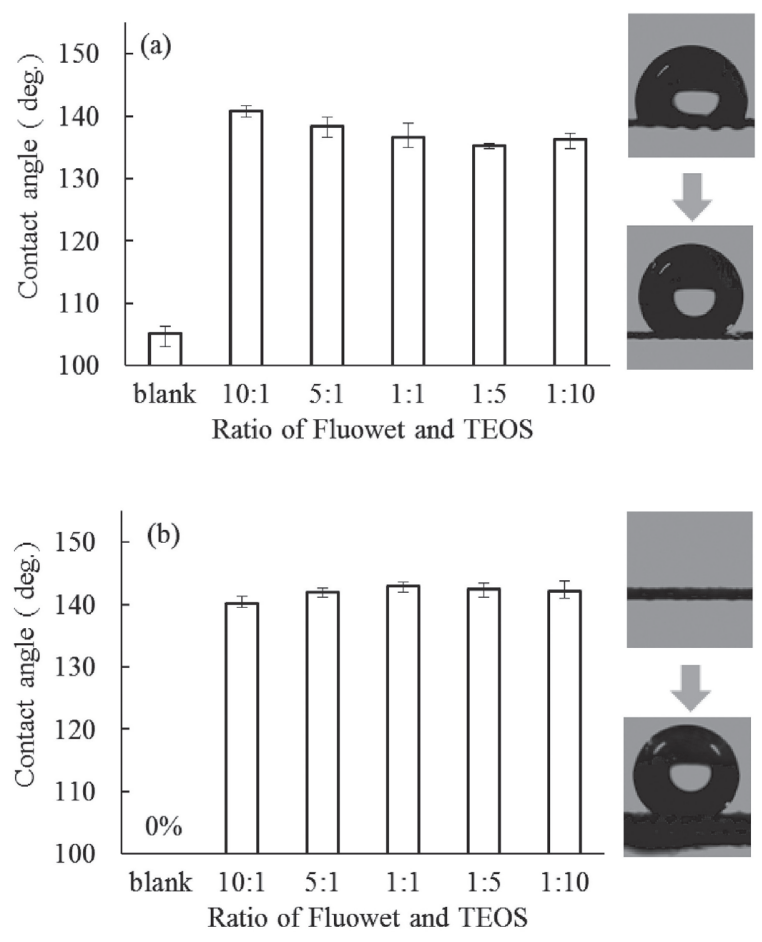

Fig. 2 Continued on next page. 

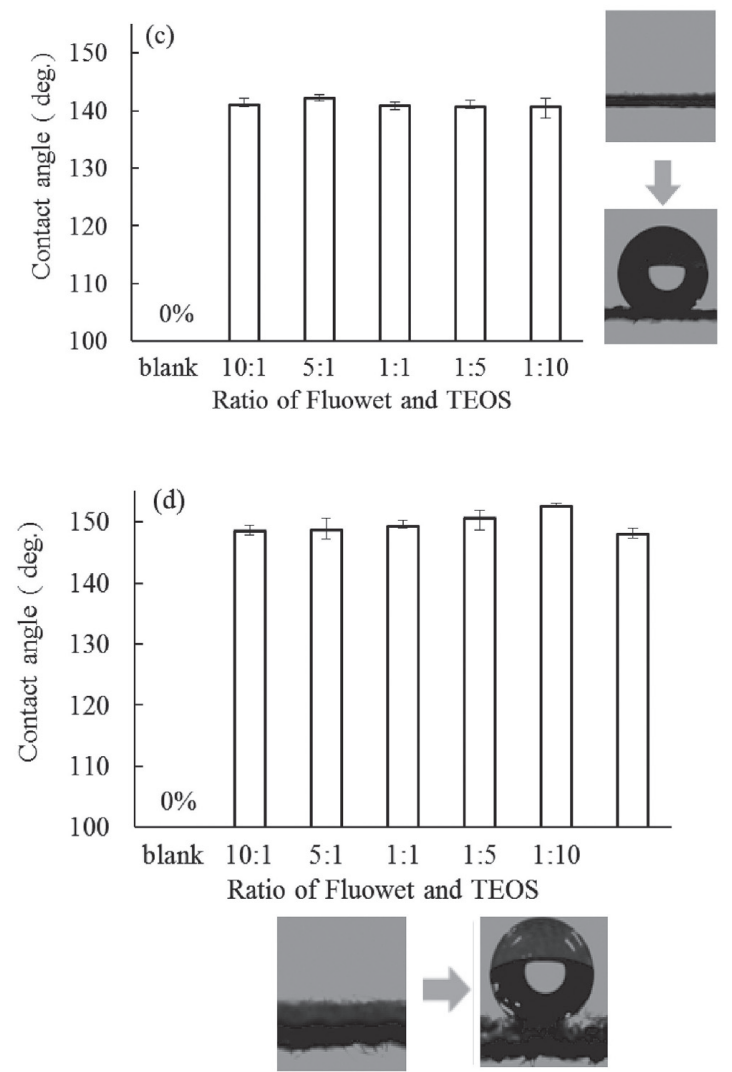

Fig. 2 Contact angle of water droplet on sol/gel treated fabrics after washing: (a) PET, (b) cotton, (c) PET/cotton and (d) Vinylon. Pictures show the observed shape of the water droplets before and after treatment.

water droplet was immersed immediately into the fabrics, so the contact angle of these fabrics blank samples were estimated to $0^{\circ}$. The mixing ratio of Fluowet and TEOS was changed from $10: 1$ to $1: 10$ (also $1: 100$ for vinylon), but the influence of the Fluowet content was very small. This means that only a small amount (ca. $10 \%$ ) of Fluowet is enough to get the sufficient water repellent treatments. In the cases of cotton and PET/ cotton higher contact angle than $140^{\circ}$ was observed in every mixture, while that in the case of PET the contact angle of water was a little smaller than $140^{\circ}$. In contrary, very high contact angle $\sim 150^{\circ}$ was observed in the case of vinylon and sufficiently high water repellent treatment

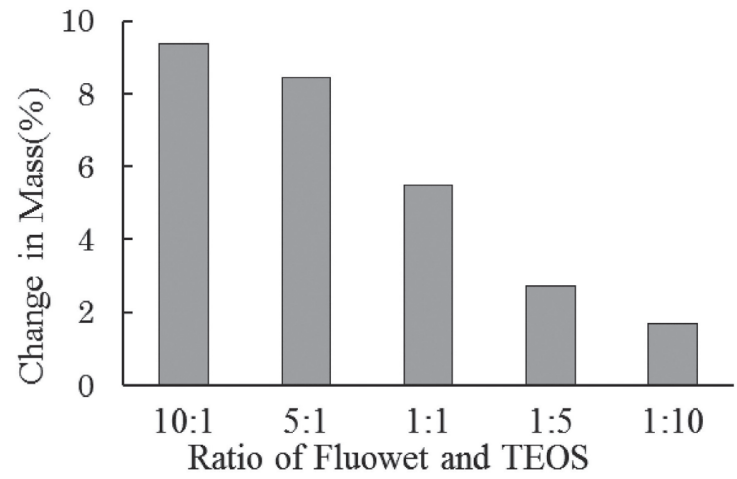

Fig. 3 Weight increment after sol-gel treatment of cotton/PET fabric (after washing).

can be done using only $1 \%$ Fluowet content.

The increment in the weight of fabric after sol/gel treatment was determined by weighting method. A typical data was shown in Fig. 3 in the case of PET/cotton fabric. The similar results were obtained in the other fabrics PET, cotton and vinylon.

The total weight was increased by increasing the content of Fluowet. However, the fabric treated with high content of Fluowet become a little harder.

\subsection{EDX analysis of surface of sol-gel treated fabrics}

EDX spectra of cotton treated by sol/gel method were shown with untreated in Fig. 4. On the surface of untreated cotton fabric no F and $\mathrm{Si}$ atoms were detected, if a noise is taken into consideration (a), while relatively large peaks were found after sol/gel treatment (b). These peaks became a little smaller after 5 times washing, but it is clear that the significant amount of Fluowet remains after strong washing (c).

Similar results were obtained in every fabric. The atomic content of $\mathrm{C}, \mathrm{O}, \mathrm{F}$ and $\mathrm{Si}$ atoms are summarized in Table 1. In every cases, the content of $\mathrm{F}$ and $\mathrm{Si}$ atoms increased significantly after sol/gel treatment and these values decreased more or less after 5 times washing. This decrement shows that not all of Fluowet molecules react with the fabrics, but the decrement was relatively small. It means that the most of Fluowet molecules reacted very hardly with the fabrics.
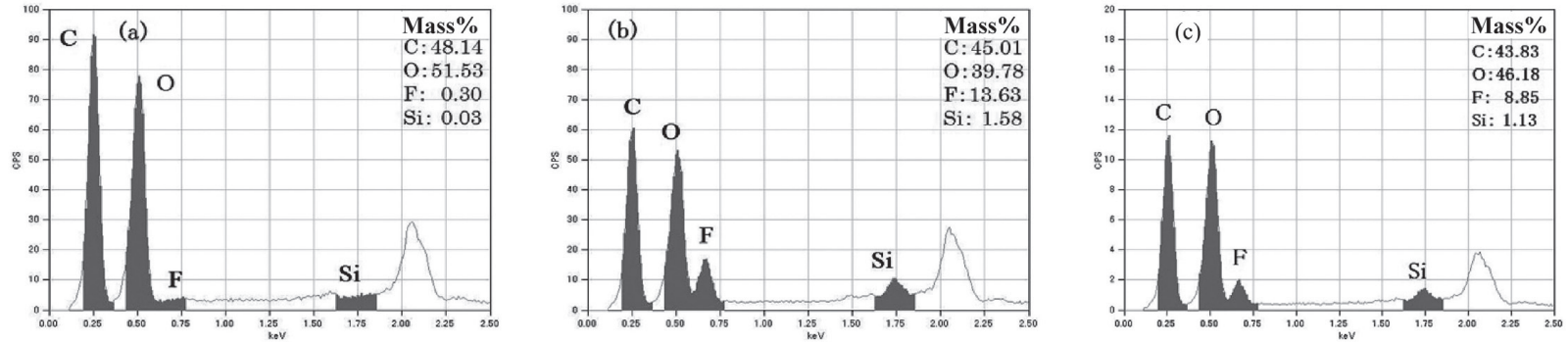

Fig. 4 EDX spectra of cotton fabric, (a) untreated, (b) sol/gel treated and (c) sol/gel treat and washed. 
Table 1 The atomic content of C, O, F and Si atoms of fabrics treated by sol-gel method.

\begin{tabular}{clrrrr}
\hline \multirow{2}{*}{ Fabric } & \multirow{2}{*}{ Treatment } & \multicolumn{4}{c}{ Atomic content \% } \\
& & $\mathrm{C}$ & $\mathrm{O}$ & \multicolumn{1}{c}{$\mathrm{F}$} & $\mathrm{Si}$ \\
\hline \multirow{3}{*}{ PET } & Blank & 64.4 & 34.4 & 0.1 & 0.0 \\
& Sol-gel only & 62.9 & 32.9 & 3.8 & 0.4 \\
& Sol-gel and washing & 63.0 & 33.9 & 2.8 & 0.3 \\
\hline \multirow{4}{*}{ PET/cotton } & Blank & 56.4 & 42.8 & 0.7 & 0.0 \\
& Sol-gel only & 53.2 & 38.8 & 7.1 & 0.8 \\
& Sol-gel and washing & 53.4 & 39.7 & 6.2 & 0.7 \\
\hline \multirow{5}{*}{ cotton } & Blank & 48.1 & 51.5 & 0.3 & 0.0 \\
& Sol-gel only & 45.0 & 39.8 & 13.6 & 1.6 \\
& Sol-gel and washing & 43.8 & 46.2 & 8.9 & 1.1 \\
\hline \multirow{5}{*}{ vinylon } & Blank & 61.6 & 40.0 & 0.3 & 0.1 \\
& Sol-gel only & 59.0 & 37.2 & 4.4 & 0.5 \\
& Sol-gel and washing & 59.7 & 35.8 & 3.9 & 0.7 \\
\hline
\end{tabular}

Infrared analysis of fiber surface was conducted using the attenuated total reflectance (ATR) attachment with a KRS-5 prism. Its spectra of cotton treated by sol/ gel method were shown in Fig. 5. Characteristic peak based on C-F stretching vibration $[15,16]$ was observed 1200 and $1240 \mathrm{~cm}^{-1}$ for sol/gel treated fabric and its washed fabric, while no peak was observed for blank cotton.

It is well known that TEOS is hydrolyzed at acidic condition to produce hydroxyl silane compounds, which can react easily with the other hydroxyl silane compounds by condensation reaction, shown in Fig. 6 .

In the present study there are two silane compounds TEOS and Fluowet in the mixture. Both compounds can react together by the similar reaction like in the right side of Fig. 6. During the agitation of mixture of Fluowet and TEOS such condensation reaction can be considered to occur and relatively high molecules condensates are produced. When the condensates are added on the fabrics and heated to high temperature, they react with $-\mathrm{OH}$

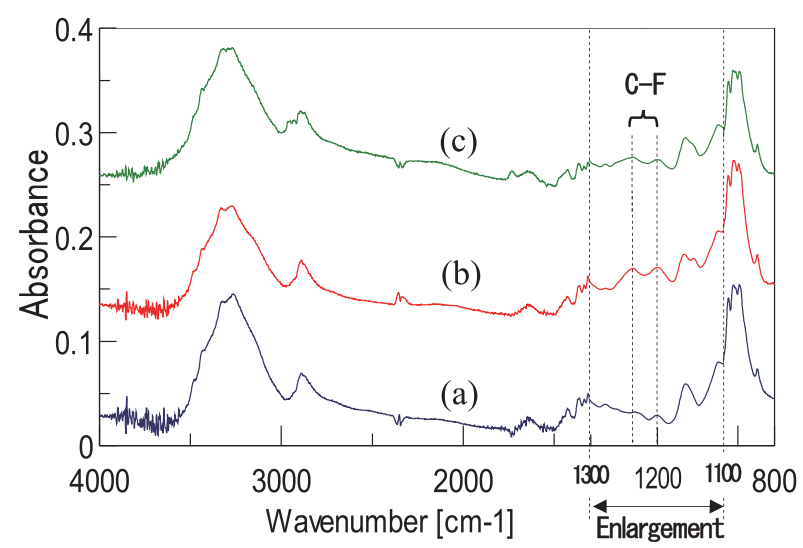

Fig. 5 ATR spectra of sol-gel treated cotton fabric. From bottom to top, (a) untreated, (b) sol/gel treated and (c) sol/gel and washed, respectively.

groups of the fabrics.

In the case of PET, the possible reason of relatively low contact angle may be attributed the extremely small amounts of reactive - $\mathrm{OH}$ end group. In contrary, for very high contact angle of vinylon, this may be explained by the fact that possesses quite many - $\mathrm{OH}$ groups to react with silane compounds and these silanol compounds were reacted by covalent binding. Similar reaction was observed in the case of Nylon fabric by Nakazumi and his coworkers [17].

\section{Summary}

A new water-repellent treatment of various kinds of fabrics having hydroxyl groups has been developed by using mixtures of tetraethoxysilane and a commercial silane compound having fluorocarbone chain, Fluowet FS 600. A small amount of Fluowet is enough to obtain high water-repellent property. The contact angle of water droplet on the treated fabrics was over $140^{\circ}$ in every fabric even after sufficient washing. The reaction was

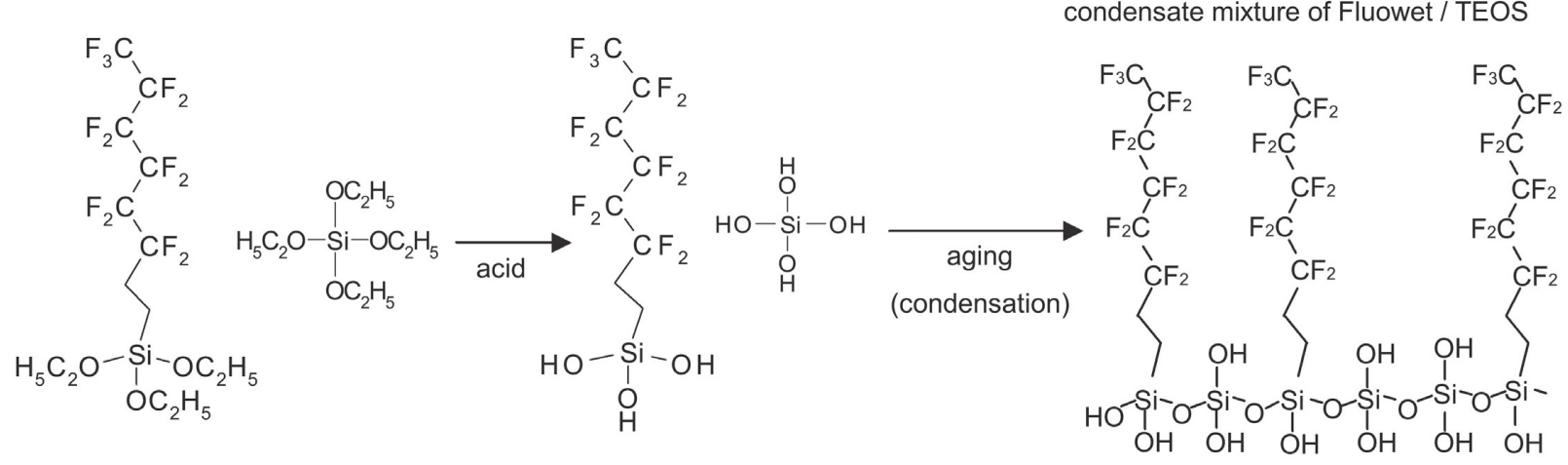

Fig. 6 Reaction scheme of TEOS and Fluowet in acidic condition. The first reaction is hydrolysis of TEOS and Fluowet, and the second reaction is condensation of hydrolyzed TEOS and Fluowet. The condensation can proceed further. 
confirmed by means of FT-IR and EDX analysis.

\section{References}

1. S. Sakka, "Development of Fluorine Materials" (Japanese), CMC, Tokyo(1997).

2. Asahi glass Co., Ltd., Kakou-Gijutsu, 46, 31 (2011).

3. M .Yamabe, J. Matsuo, "Development of fluorinated materials", CMC, Tokyo(1997).

4. M. Lee, K. Nishi, D. S. Jeong, T. Tokuyama, T. Itazu, Y. Miyaji and T. Wakida, Sen 'i Gakkaishi, 61, 309 (2005).

5. K. Nakamae : Engineering materials, 44, 26 (1996).

6. A. Nakajima, K. Hashimoto and T. Watanabe, Monatsh. Chem., 31, 132 (2001).

7. A. Vilcnik, I. Jerman, A. S. Vuk, M. Kozelj, B. Orel, B. Tomsic, B. Simoncic and J. Kovac, Langmuir, 25, 5869 (2009).

8. X. Peng, T. Kawase, M. Sato, K. Ikeno and H. Sawada, Sen'i Gakkaishi, 58, 91 (2002).
9. T. Yasui, Master Dissertation, Fukui University (2001).

10. K. Teshima, H. Sugimura, Y. Inoue, O. Takai, and A. Takano, Langmuir, 19, 10624 (2003).

11. H. Wang, J. Fang, T. Cheng, J. Ding, L. Qu, L. Dai, X. Wanga and T. Lin, Royal Soc. Chem., 877 (2008).

12. T. Ogawa, B. Ding, Y. Sone and S. Shiratori, Nanotechnology, 18, 165607 (2007).

13. Z.-Z. Gu, H. Uetsuka, K. Takahashi, R. Nakajima, H. Onishi, A. Fujishima and O. Sato, Angew. Chem., Int. Ed., 42, 894 (2003).

14. S. Sakka, "Application of Sol-Gel Method" (Japanese), Agne Shofusha, Tokyo (1997).

15. Y. Han, J. Lin and H. Zhang, Materials Letter, 389 (2002).

16. Y. Yu, M.Z. Rong and M.Q. Zhang, Polymer, 51, 492 (2010).

17. K. Satoh, H. Nakazumi, Textile Res. J. 74, 1081 (2004). 